\title{
Vibrational damping behaviors of graphene nanoplatelets reinforced NR/EPDM nanocomposites
}

\author{
N. Mohamad ${ }^{1 *}$, J. Yaakub ${ }^{1}$, H.E. Ab Maulod ${ }^{1}$, A.R. Jeefferie ${ }^{1}$, M.Y. Yuhazri ${ }^{1}$, \\ K. T. Lau' ${ }^{1}$ Q. Ahsan ${ }^{1}$, M.I. Shueb ${ }^{2}$ and R. Othman ${ }^{1}$ \\ ${ }^{1}$ Carbon Research Technology, Advanced Manufacturing Centre, Faculty of \\ Manufacturing Engineering, Universiti Teknikal Malaysia Melaka, Hang Tuah Jaya, \\ 76100, Durian Tunggal, Melaka, Malaysia \\ *Email: noraiham@utem.edu.my \\ Phone: +6063316976; Fax: +6063316411 \\ ${ }^{2}$ Composites and Polymer Blends Group, Radiation Processing Technology Division, \\ Malaysian Nuclear Agency, Bangi, 43000, Kajang, Malaysia
}

\begin{abstract}
Vibrational damping behaviors of graphene nanoplatelets (GNPs) reinforced natural rubber/ethylene-propylene-diene rubber (NR/EPDM) were investigated. The nanocomposites were prepared through melt compounding using a Haake internal mixer. In this study, GNPs reinforced NR/EPDM nanocomposites were characterized for damping properties towards the effect of GNPs loading (1, 3 and $5 \mathrm{wt} \%)$ and chitosanfunctionalization. The results indicate the addition of GNPs have significantly improved both $\log$ decrement and damping ratio values correlated to the enhanced damping property of the nanocomposites. Chitosan-functionalized GNPs seem to slightly hamper the increase of damping properties contributed by incorporation of GNPs in NR/EPDM blends. These findings were further supported by thermal and morphological studies through dynamic mechanical analysis (DMA) and transmission electron microscopy (TEM) analysis. The storage modulus, loss modulus and tan $\delta$ showed good agreement with the vibrational damping test. Meanwhile, TEM analysis revealed the existence of intercalated and exfoliated structure of GNPs in NR/EPDM matrix which resulted in improved vibration damping properties of the nanocomposites for the potential used as vibration insulator.
\end{abstract}

Keywords: Graphene; NR/EPDM; vibration; dynamic mechanical; morphology.

\section{INTRODUCTION}

Rubbers have been extensively used as a vibration isolator or dampers due to their viscoelastic properties [1]. It has become one of the most important elastomeric material for energy absorption and most importantly, is a key component of many load-bearing structures and vibration-isolation mountings. To date, there are widespread use of rubber components for noise and vibration isolation in aerospace and automotive industries [23]. This is agreed by Geisberger et al. [4] which stated the significant role of rubber compound in developing novel materials for reducing undesirable noise and preventing vibration fatigue failure. Outstanding damping characteristics in rubbers owing to the transition of mechanical vibration to thermal energy through friction between molecular chains and heat dissipation [5]. The major constituents of a typical elastomer compound 
are long chain molecules known as the base polymer which provide the basic chemical and physical characteristics. A small amount of free space exists between the long chain molecules which allows for the movement of the elastomeric molecules independently of one another to deform and change shapes. Crosslinks formed within the closely packed molecular network during vulcanization and the addition of fillers influence the reversibility of elastomers. Vulcanized natural rubber (NR) has been extensively used in the application of static and dynamic applications due to its excellent properties such as high elasticity and toughness [6]. However, single NR nanocomposite has poor thermal and ozone properties. Blending of two or more polymers produces material with improved properties, better processability and making it superior than its original phases at minimal cost [7]. For instance, rubber blends are widely utilized in automobile parts such as springs and snubbers, hoses and belting, seals and bearings, engine mounts, gear gaskets, pneumatic tires and tubes. For the past few years, many researchers have integrated NR and EPDM synthetic rubber for the preparation of elastomeric blends as stated by Mohamad et al. [8]. NR/EPDM has exciting potential in outdoor applications because of its excellent thermal, chemical and ozone resistance, as well as attractive dynamic properties. The poor outdoor properties of high unsaturated NR can be significantly improved by the incorporation of EPDM without sacrificing their unique mechanical properties. Vulcanized NR/EPDM blend systems have been extensively studied because of their superior performance in tire application as well as significant improvement in heat and ozone resistance [9-10]. In the case of blending two incompatible rubber phases like NR and EPDM, the addition of nanoparticles into the elastomeric blend is interesting and simple since it can strongly adsorb into the blend's interface and consequently able to stabilize it [11]. In addition, the presence of various functional fillers in NR/EPDM blends may enhance the engineering properties, as well as its functionalities [12]. For this study, NR/EPDM blend was incorporated with the latest and popular carbonaceous nanomaterials of graphene nanoplatelets (GNPs). GNPs are emerging as advanced materials due to their prominent intrinsic properties. Graphene, a basic unit of GNPs is a monolayer of sp2-hybridized carbon atoms arranged in a twodimensional lattice. It has a large surface area and tunable surface properties with Young's modulus of $0.5-1 \mathrm{TPa}$, a breaking strength of around $42 \mathrm{Nm}^{-1}$, an ultimate strength of $130 \mathrm{GPa}$, as well as high thermal conductivity of above $5000 \mathrm{WmK}^{-1}$. The platelet thickness is in the range of $0.3-100 \mathrm{~nm}$, while the density of GNPs is $\sim 2 \mathrm{gcm}^{-3}$ [13]. The high aspect ratio combined with the extraordinary properties of GNPs thus make them as an ideal reinforcing and functional fillers for polymer nanocomposites. The large surface area of GNPs increases the interface contact between the platelets and matrices that could be beneficial for interfacial polarization. The downsides, graphene materials are unsuitable for intercalation by large species, such as polymer chains. The graphene as a bulk material has a pronounced tendency to agglomerate in a polymer matrix hence, various functionalization or surface treatment are conducted by various researchers. According to Kuila et al. [14], oxidation proceeded by chemical functionalization will facilitate the dispersion and stabilize the graphene from agglomerations. In fact, functionalization and dispersion of GNPs are significance in their applications. Proper chemical functionalization of graphene also prevents restacking and agglomerations of individual graphene sheets as well as retaining their inherently excellent properties [15]. There are two major approaches for obtaining well-dispersed GNPs either through covalent or noncovalent functionalization. This functionalization relies on whether the GNPs covalently or noncovalently bonded with the functional groups or modifier molecules during the treatment process. In addition, it has been pointed out that each type 
of surface modification has its own merit to improve the interfacial interaction between graphene and polymer matrix [16].

The dispersion of GNPs must be done judiciously to fully exploit its extraordinary mechanical, thermal and electrical properties. Its delicate morphological organization, fine interface control, uniform dispersion and ease of processing are vital to the performance of the resulting composites [17]. In polymer nanocomposites, nanofillers dispersion as well as interfacial interaction has been shown to be an important parameter for the enhancement of mechanical properties [18]. In fact, according to Patole et al. [19], poor dispersion of graphene nanoplatelets (GNPs) is one of the biggest challenges involved while forming the composite materials in bulk amounts because of the hydrophobic nature, strong $\pi-\pi$ stacking and the strong Van de Waals force that holds the nanoplatelets together. Even after the initial process of exfoliation and dispersion, these strong interactions often lead to significant aggregation or agglomeration thus destroys their unique properties of the individual graphene sheet. Therefore, like any other nanomaterials, preventing GNPs from agglomerations are important to assure the exceptional properties of individual graphene's sheet can be preserved. Chitosan, a natural polysaccharide has been used as polymer dispersant of GNPs. due to their excellent biocompatibility, biodegradability and binding ability [20]. Moreover, chitosan is a stimuli-responsive polymer that can change its form reversibly when alerted by $\mathrm{pH}$ changes [21]. The presence of chitosan for dispersing GNPs can lead to an interesting system that could possibly combine both merits of GNPs and chitosan. Noncovalent functionalization of GNPs with chitosan performed in this study was based on the study by Ganguli et al. [22] and Fang et al. [23]. It is hypothesized that chitosan could potentially provide GNPs with both biocompatibility and the efficacy of treatment work. While there is a number of researchers who pay more attention on mechanical properties of graphene-based polymer nanocomposites, there have been relatively fewer attempts currently to investigate vibration damping behavior of nanoparticle reinforced rubber [24]. To the best of our knowledge, there is scarcity of study on vibrational damping behaviors of graphene based elastomeric nanocomposites. In fact, there is an immense potential to develop rubber nanocomposites with ideal vibration damping capacity with incorporation of GNPs. Previously, we had optimized the process parameters for melt compounding of NR/EPDM filled GNPs nanocomposites as well as investigated the effect of chemical functionalization on GNPs [8-9,13,25]. In this present study, chitosanfunctionalized and unfunctionalized GNPs at different loadings were characterized for damping behavior in terms of their log decrement and damping ratio values in free vibration test. These findings were further supported by thermal and morphological data through DMA and TEM analyses.

\section{METHODS AND MATERIALS}

\section{Materials}

The nanocomposite formulation used in the present study is given in Table 1. Natural rubber, SMR 20 grade was supplied by Malaysian Rubber Board. Ethylene propylene diene monomer used was EPDM Buna ${ }^{\circledR}$ EPT 9650 procured from Lanxess Corp. with ENB content of $6.5 \pm 1.1 \mathrm{wt}$. \%; ethylene content of $53 \pm 4 \mathrm{wt} \%$ and Mooney viscosity UML $(1+8)$ at $150^{\circ} \mathrm{C}$ of $60 \pm 6$. Epoxidized natural rubber (ENR) under the trade name of ENR-50 with 53\% epoxidization was supplied by Malaysian Rubber Board. The average Mooney viscosity measured at $\mathrm{ML}(1 \pm 4) 100^{\circ} \mathrm{C}$ was 67 , and the average specific 
gravity at approximately $25^{\circ} \mathrm{C}$ was 0.9366 . It was used as a compatibilizer to increase the miscibility between NR and EPDM. Other compounding ingredients such as sulphur, zinc oxide, and stearic acid were purchased from System/Classic Chemical Sdn Bhd; tetramethyl thiuram disulfide (Perkacit-TMTD) and 2,20-dithiobis(benzothiazole) (Perkacit-MBTS) was acquired from Perkacit; N-(1,3-dimethylbutyl)- N'-phenyl-pphenylenediamine (6PPD) were supplied by Flexsys America (United States). Commercialized graphene nanoplatelets, GNPs KNG-150 was supplied by Xiamen Graphene Technology Co. Ltd, China with true density of $\sim 2.25 \mathrm{~g} / \mathrm{cm}^{3}$ and carbon content of $>99.5 \mathrm{wt} \%$. The designation of samples for this study is tabulated in Table 2.

Table 1. Typical formulations.

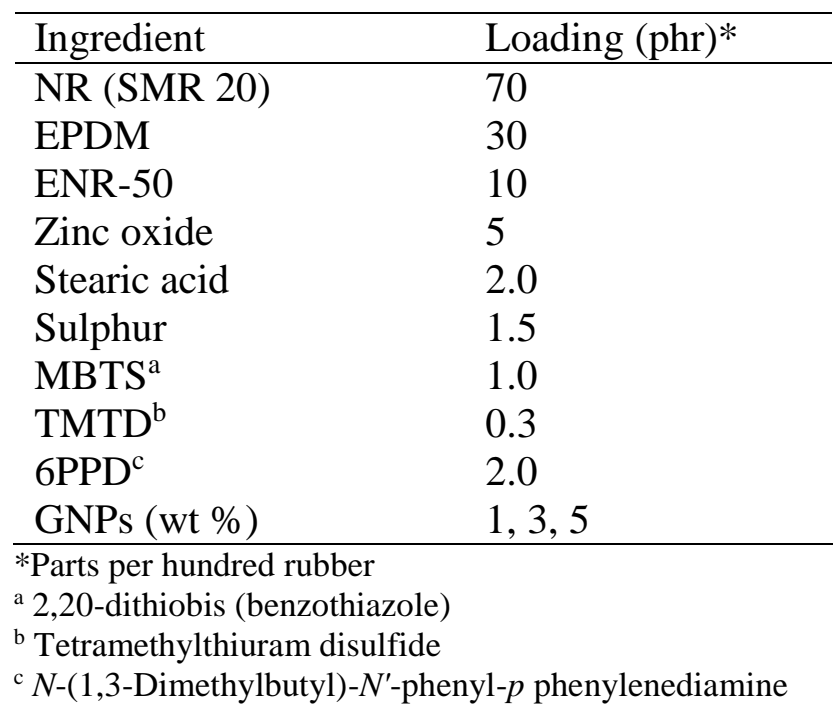

\section{Sample Preparation of GNPs reinforced NR/EPDM}

For chitosan-functionalized GNPs, the GNPs were firstly ultrasonicated in a mixture of water/ethanol for 2 hours. The GNPs dispersion was then gradually added into an acidic aqueous solution of chitosan $(3 \mathrm{~g}$ in $0.5 \mathrm{M} \mathrm{HCl})$ by applying ultrasound for another 2 hours to yield a uniform black-colored dispersion. The dispersion was then filtered through a Millipore hydrophilic $(0.22 \mu)$ filter paper and washed with distilled water to eliminate unreacted chemicals. Then, the chitosan-functionalized GNPs were dried in a vacuum oven at $70^{\circ} \mathrm{C}$ for 24 hours. Finally, the chitosan-functionalized GNPs was ground and placed in a sealed container before nanocomposites preparation. Meanwhile, for unfunctionalized GNPs, the GNPs undergone sonication in mixture of water/ethanol for 4 hours, filtered, washed and dried in oven. GNPs reinforced NR/EPDM was prepared via melt compounding using a Haake internal mixer at mixing temperature of $110^{\circ} \mathrm{C}$, rotor speed of $40 \mathrm{rpm}$ and mixing time of $7 \mathrm{~min}$. Firstly, NR, EPDM and ENR-50 were masticated for $1 \mathrm{~min}$ before the rest of ingredients and GNPs (unfunctionalized or functionalized) were simultaneously added except for the curative agents. Finally, sulphur and accelerators were added and mixed for about $1 \mathrm{~min}$ before the mixture was dumped and left to cool to room temperature. From this stock, sheets approximately $2 \mathrm{~mm}$ thickness were vulcanized with a semi-efficient vulcanization system with a hot press at $150^{\circ} \mathrm{C}$ at the respective cure times, $\mathrm{t} 90$ which was derived from a rheometer test in accordance with ASTM D 2084. 
Table 2. Designation of samples.

\begin{tabular}{lcc}
\hline \multicolumn{1}{c}{ Samples } & $\begin{array}{c}\text { GNPs Loading } \\
(\mathrm{wt} \%)\end{array}$ & $\begin{array}{c}\text { Chitosan } \\
\text { Functionalization }\end{array}$ \\
\hline $\begin{array}{l}\text { Unreinforced vulcanized NR/EPDM } \\
\text { (R1) }\end{array}$ & 0 & Without \\
\hline NR/EPDM $+5 \mathrm{wt} \%$ f-GNPs (R2) & 5 & With \\
\hline NR/EPDM $+1 \mathrm{wt} \%$ uf-GNPs (R3) & 1 & Without \\
\hline NR/EPDM + 5wt\% uf-GNPs (R4) & 5 & Without \\
\hline NR/EPDM + 3wt\% uf-GNPs (R5) & 3 & Without \\
\hline
\end{tabular}

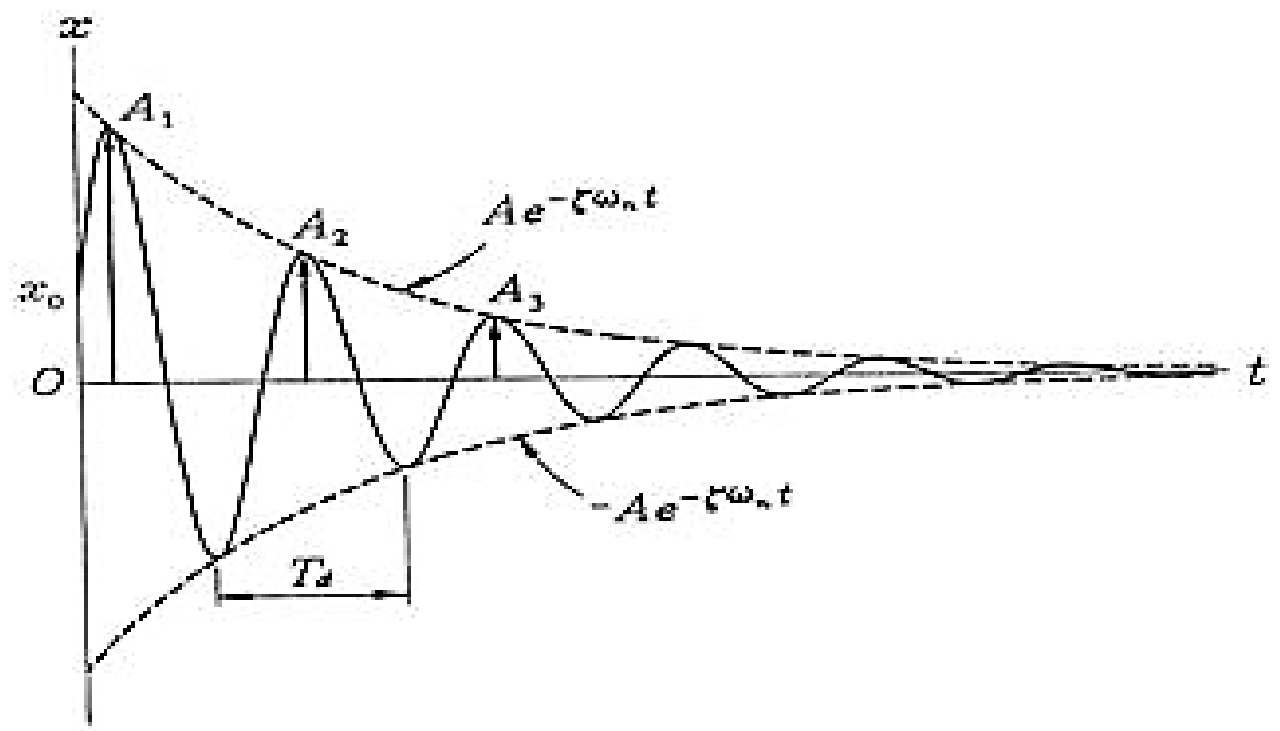

Figure 1. Logarithmic decrement method (free vibration of a damped single degree of freedom system).

\section{Accelerometer (Free Vibration Test) - Logarithmic Decrement Method}

Damped vibration via second order dynamic system is described in terms of the undamped natural frequency, $\omega_{\mathrm{n}}$ and the damping ratio, $\zeta$. A typical free response can be modelled by the expression in Eq. (1):

$$
x(t)=A e^{-\zeta \omega n t} \cos (\omega d t-\varphi)
$$

A and $\varphi$ are the peak amplitude and phase angle, as determined by the initial conditions. By referring to the plot of damped oscillations as in Figure 1, the damped natural frequency, $\omega_{d}$ can be found from the inverse of the oscillation period:

$$
\omega_{d}=2 \pi f=\frac{2 \pi}{\tau_{d}}
$$

where $T_{d}$ is the damped oscillation period. The damped natural frequency is also found by:

$$
\omega_{d}=\omega_{f} \sqrt{1-\zeta^{2}}
$$


$\omega_{\mathrm{n}}$ is undamped natural frequency. Since the cosine function is one at those maximum points, it gives:

$$
x\left(t_{k}\right)=A e^{-\zeta \omega n t k}
$$

Thus, the envelope of the vibration decays in a manner that looks exactly like a first-order system. Then, the time between successive oscillation cycles is given by:

$$
t_{k+1}-t_{k}=\frac{2 \pi}{\omega}
$$

which provides the fractional amount of amplitude reduction that can be seen after one cycle of motion has elapsed. After $\mathrm{N}$ cycles:

$$
\frac{x\left(t_{k+1}\right)}{x\left(t_{k}\right)}=\exp \left(-\frac{2 \pi \zeta N}{\sqrt{1-\zeta^{2}}}\right)
$$

Therefore, the log-decrement is shown as

Then damping ratio for the system is

$$
\delta=\frac{1}{N} \ln \left(\frac{x\left(t_{k}\right)}{x\left(t_{k+1}\right)}\right)
$$

$$
\zeta=\sqrt{\frac{\delta^{2}}{(2 \pi)^{2}+\delta^{2}}}
$$

\section{Measurements of Vibration Damping Properties}

Rectangular specimens of $30 \mathrm{~mm}$ wide $\mathrm{x} 80 \mathrm{~mm}$ long x $2 \mathrm{~mm}$ thick were cut from the vulcanized NR/EPDM nanocomposites. The specimens were tested in free vibration modes and the corresponding configurations are given in Figure 2. Figure 3 shows the experimental set-up that consists of accelerometer, photon analyzer, shaker, fixture, amplifier and filter are used. The free sample length is defined as the distance between the clamp (fixture) and the sample tip. In free vibration mode, sample was tightly fixed at preferably $65 \mathrm{~mm}$ from the free end deflected to a desired displacement before release. The accelerometer was connected to the input jack of a second power unit using a microdot-BNC cable. The output jack of the power unit was then connected to Channel 4 of the DAQ system using a BNC cable. Then, accelerometer was attached to the tip of the specimen and both the power units and DAQ system were turned on. The resulting vibration response was continuously monitored using the accelerometer attached to the tip of the specimen.

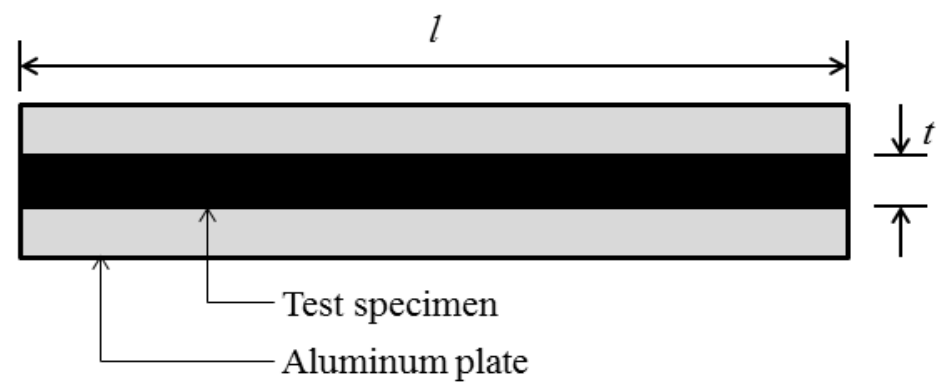

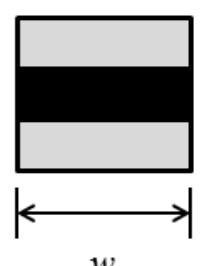

Figure 2. Shape and dimensions of test specimen for the measurement of the vibration damping properties. 


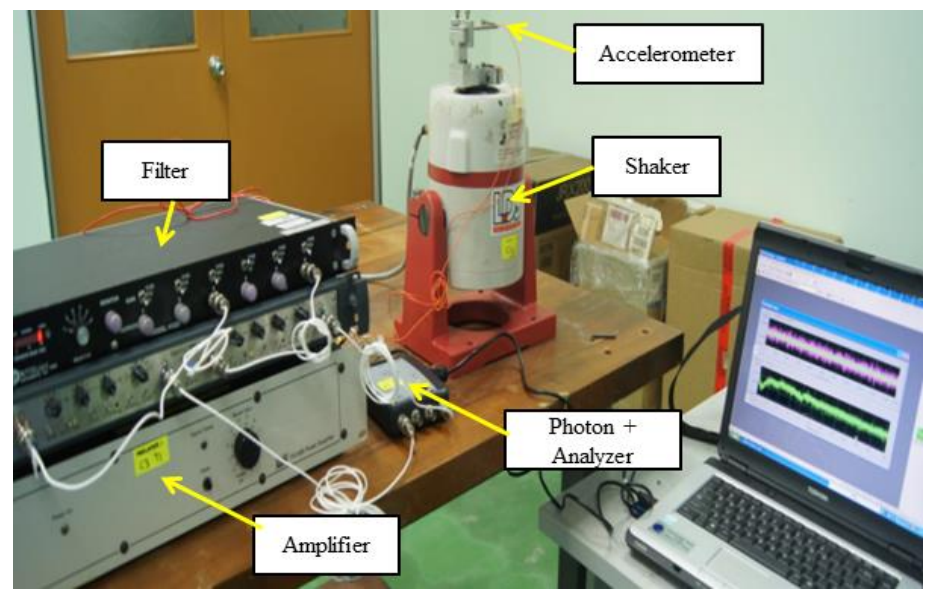

Figure 3. Test set-ups for free vibration test.

\section{Dynamic Mechanical Analysis}

Dynamic mechanical analysis was performed using a Perkin-Elmer DMA-7e analyzer (U.S.A.) to assess the dynamic-mechanical performance of the NR/EPDM nanocomposites prepared. The measurement was carried out at heating rate of $10^{\circ} \mathrm{C} \mathrm{min-}$ lover a temperature range of -80 to $40{ }^{\circ} \mathrm{C}$.

\section{Transmission Electron Microscopy}

Transmission electron microscopy (TEM) allows a qualitative understanding of the structure of the nanocomposites through direct observation. It enables to identify the morphology of the nanocomposites and to show if the graphene nanoplatelets are welldispersed or not with high magnification images. Ultrathin sections with thickness of approximately $80 \mathrm{~nm}$ were cut with Leica Ultracut UCT ultra microtome cooled to $-100^{\circ} \mathrm{C}$ using liquid nitrogen and placed onto copper grid. Later the samples were observed under TEM using a voltage of $200 \mathrm{Kv}$.

\section{RESULTS AND DISCUSSION}

\section{Vibration Test (Free Vibration Mode- Logarithmic Decrement Method)}

Analysis on vibration damping properties provides the information on dynamic characteristics of a material under real operation condition. According to Khan et al. [26], crosslinks within the closely packed network formed during vulcanization and interfacial interaction between added nanofillers within polymer matrix influence the damping capacity of a polymer composite. Figure 4 shows the log decrement curves vs time history of NR/EPDM blend and NR/EPDM nanocomposites at various GNPs loading (unfunctionalized and functionalized). In Figure 4, the amplitude of R4 and R5 samples decays faster than vulcanized NR/EPDM blends (R1) and other nanocomposites; R2 and R3 samples. Thus, the experiment proved the efficiency of GNPs reinforcement to NR/EPDM blend without chitosan-functionalization. The amplitude of vibration for unfunctionalized samples at 5 and $3 \mathrm{wt} \%$ GNPs (R4 and R5) were observed to decay much faster than chitosan-functionalized sample (R2) and control sample without GNPs (R1). After a period of 0.1 seconds, the amplitudes of R4 and R5 samples were reduced by $14.3 \%$ and $28.3 \%$ respectively. The log decrement test confirmed that the damping ratio significantly improved with the presence of GNPs and the enhancement was the highest by incorporating $3 \mathrm{wt} \%$ unfunctionalized GNPs (R5). This is implying a 
significant amount of GNPs reinforcement is crucial to increase the dissipation of energy in NR/EPDM structure. This was due to extremely high specific surface area (surface area to mass ratio) of GNPs had consequently produced closer contact with the NR/EPDM matrix. Thus, the increase in interfacial friction between the graphene sheets and the NR/EPDM matrix enhanced the energy dissipation ability. Hence, it can be concluded that the addition of GNPs in NR/EPDM nanocomposites had significantly improves its dynamic mechanical properties; damping ratio and natural frequency.

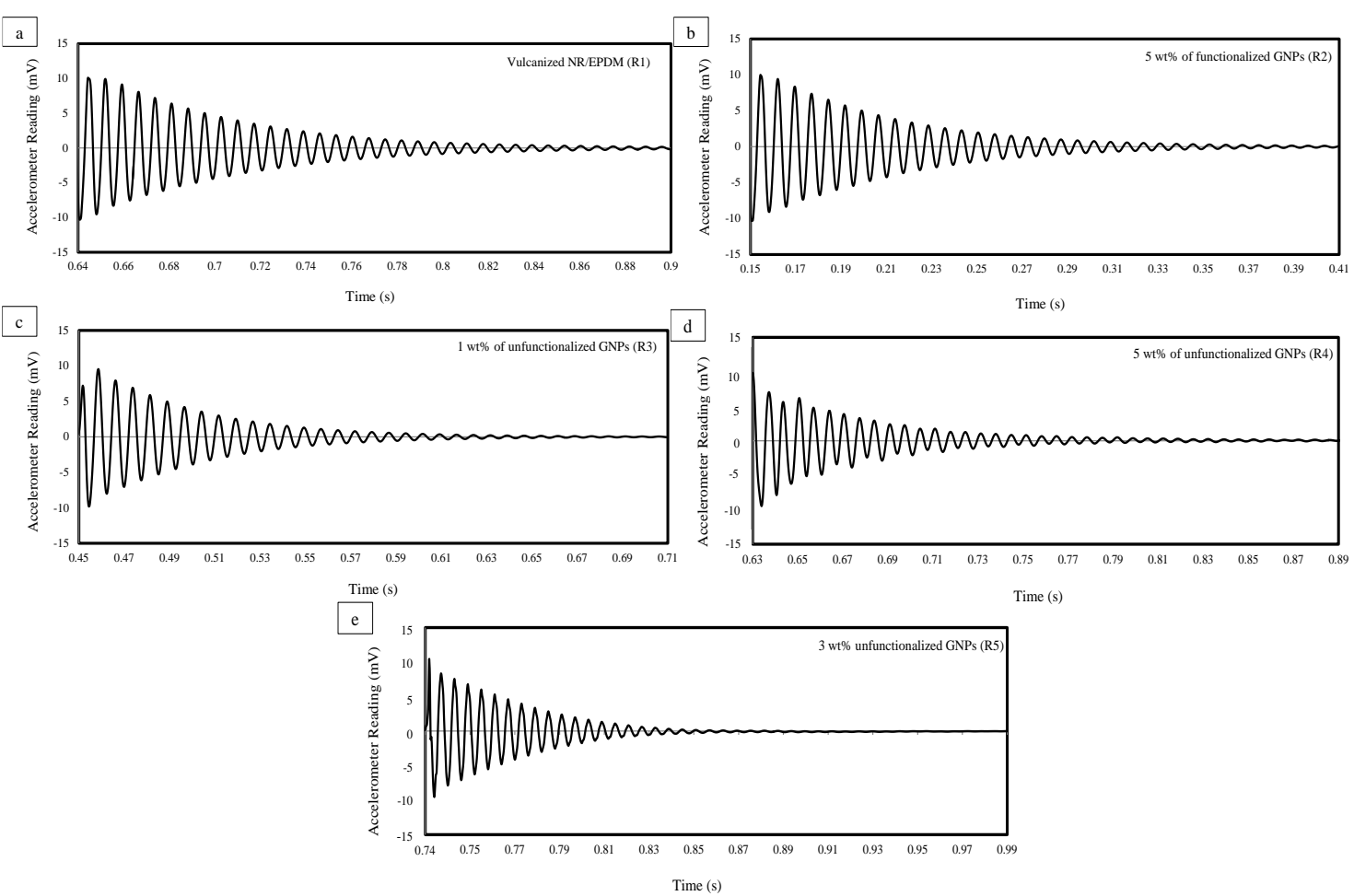

Figure 4. Free decay curves of GNPs reinforced NR/EPDM nanocomposites containing (a) unreinforced vulcanized NR/EPDM (R1); (b) 5wt \% functionalized GNPs (R2); (c) $1 \mathrm{wt} \%$ unfunctionalized GNPs (R3); (d) $5 \mathrm{wt} \%$ unfunctionalized GNPs (R4) and (e) 3wt $\%$ unfunctionalized GNPs (R5).

The presence of GNPs enhanced the logarithmic decrement and damping ratio of the NR/EPDM blends. The damping ratio is used to indicate the amount of damping in a system and it was calculated per Eq. (8) based on the logarithmic decrement method presented in Figure 4. Table 3 shows the logarithmic decrement $(\delta)$ and damping ratio $(\zeta)$ of the nanocomposites. It is noted that the highest value is exhibited by nanocomposites filled with 3 wt\% unfunctionalized GNPs (R5). This was attributed to the additional energy loss (dissipated energy) resulted from the interfacial contact area of GNPs within the composites. The stronger interactions between the rubber chains and GNPs (rubberfiller interaction), the more energy was dissipated. In such condition, the amplitude of free vibration of R5 sample decayed much faster than unreinforced rubber and other samples. Thus, it showed that GNPs enhanced the energy dissipation capability and significantly improves the vibration damping property of the composite. 
Table 3. Log decrement values and damping ratios of selected GNPs reinforced NR/EPDM nanocomposites samples.

\begin{tabular}{ccc}
\hline Samples & Logarithmic decrement, $\delta$ & $\begin{array}{c}\text { Damping } \\
\text { ratio, } \zeta(\%)\end{array}$ \\
\hline Unreinforced vulcanized NR/EPDM (R1) & 0.113 & 1.80 \\
NR/EPDM + 5wt\% f-GNPs (R2) & 0.120 & 1.91 \\
NR/EPDM + 1wt\% uf-GNPs (R3) & 0.130 & 2.07 \\
NR/EPDM + 5wt\% uf-GNPs (R4) & 0.150 & 2.37 \\
NR/EPDM + 3wt\% uf-GNPs (R5) & 0.170 & 2.70 \\
\hline
\end{tabular}

From the vibration test, GNPs exhibited a positive dynamic response and better vibration damping properties than vulcanized NR/EPDM matrix. Hence, GNPs reinforced NR/EPDM composites subjected to vibration could dampen the amplitude of vibration faster than vulcanized NR/EPDM blends. Thus, it is anticipated that GNPs incorporation can improve the service life of the composite structures. Later, DMA analysis was conducted to support and reveal the dynamical behaviors response of the selected NR/EPDM nanocomposites samples from the vibration test at different frequencies.

\section{Dynamic Mechanical (DMA) Analysis}

DMA is a powerful technique for investigating the viscoelastic characteristics and relaxation processes in polymers and polymer composites [27]. It is particularly useful to evaluate the mechanical properties of viscoelastic materials for it to exhibit time, temperature and frequency dependence. Figure 5, 6 and 7 respectively represent the dynamic storage modulus ( $\left.E^{\prime}\right)$, loss modulus $\left(E^{\prime \prime}\right)$ and tan $\delta$ factor for NR/EPDM blend and NR/EPDM nanocomposites reinforced with unfunctionalized and functionalized GNPs as the function of temperature. According to Menard [28], storage modulus is a measure of maximum energy stored in a material during one cycle of oscillation. It is well noted that structural of rubber matrix is mainly behaving at three physical states which changing from glassy state, glass-rubber transition to rubbery state and vice-versa. At the glassy state, the motion of individual chain segments is mostly frozen caused by their tightly packed state which enable the material to store more energy thus, resulting in a high storage modulus. The changes vary with temperature and closely related with the rubber chain's flexibility under stresses. In Figure 5, it is observed that storage modulus decreased with temperature hence, the components became more mobile since it lost their close packing arrangement. Therefore, in rubbery region, there was no notable change in modulus which was the result of a highly-entangled state of the rubber macromolecules. The $3 \mathrm{wt} \%$ of unfunctionalized GNPs loading (R5) exhibited the highest storage modulus if compared to vulcanized control sample NR/EPDM (R1) and other nanocomposites. This is due to the stiffening effect by GNPs hindering the mobility of rubber matrix [29] and their hydrodynamic effect associated with the strong interaction between GNPs and NR/EPDM molecules. Moreover, R5 sample possessed a higher storage modulus for their high surface area resulting in the formation of more rubber-filler interactions. On the other hand, lower intermolecular interactions caused by agglomerations of GNPs in NR/EPDM matrix led to a lower storage modulus as observed in R2 sample. 


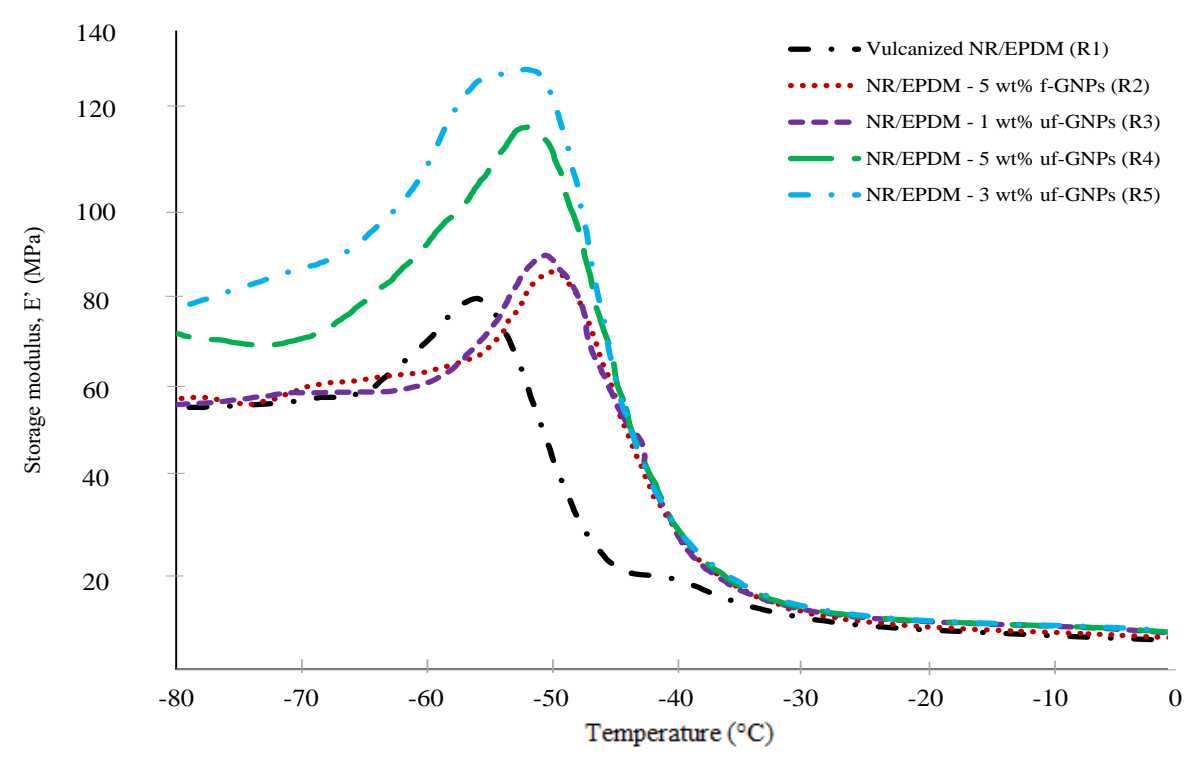

Figure 5. Storage modulus as a function of temperature.

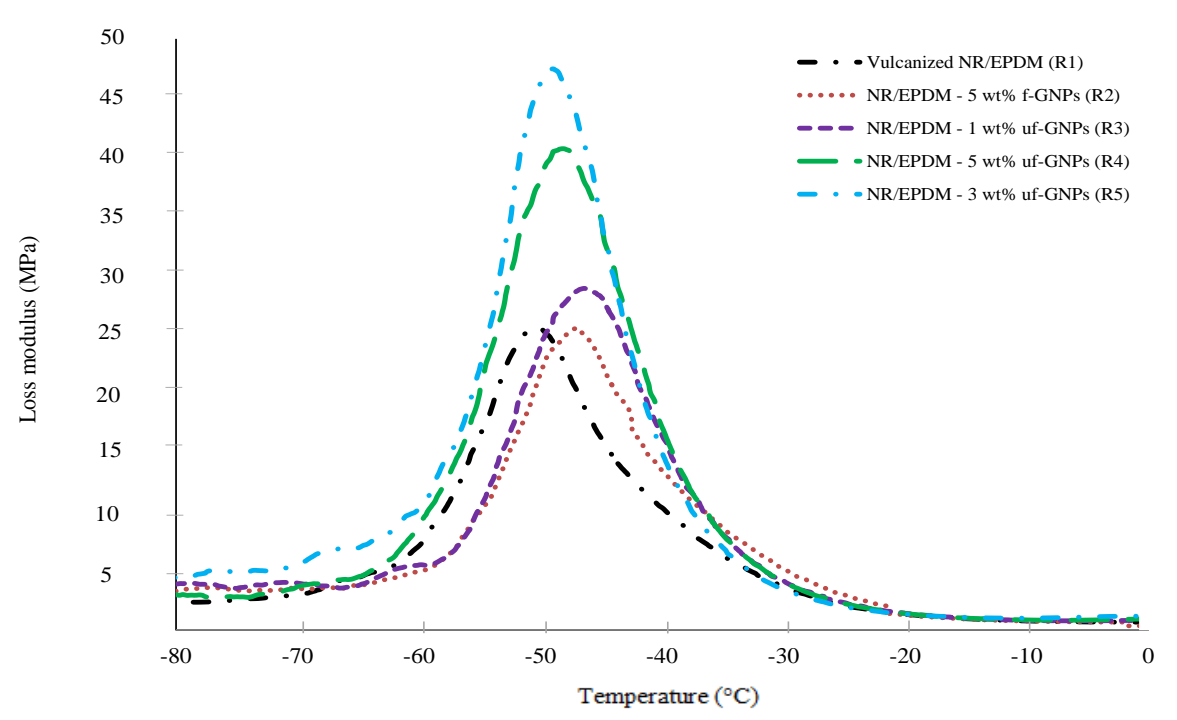

Figure 6. Loss modulus as a function of temperature.

Figure 6 demonstrates the loss modulus curves of the NR/EPDM blend and NR/EPDM nanocomposites. The loss modulus represents the energy dissipated as heat and reflects the internal motions of viscous segment per cycle under deformation. Higher amount of dissipated energy was converted into heat energy under load for NR/EPDM composites filled with unfunctionalized GNPs (R4 and R5), as shown by an increase in their loss modulus. This result indirectly indicated the improved damping performance of both samples. In contrast, the loss modulus peaks of R1 and R2 are broader due to poorer rubber-filler interaction hence reducing energy dissipation. The magnitude of damping factor $(\operatorname{Tan} \delta$ ) is indicative of a polymer system and shows the balance between elastic and viscous phase in a polymeric material [28]. It is an important parameter which represents the macromolecular viscoelasticity and the damping capacity of a material. According to Jones [30], damping is the measure of material ability to convert the mechanical energy to heat when the material is subjected to an external load. The results 
in Figure 7 confirm the better damping capacity showed by R4 and R5 than R1, R2 and $\mathrm{R} 3$ over the whole temperature range where Tan $\delta$ for R 4 and R5 were higher compared to the other samples. This was attributed to the added effect of higher absorption energy related to internal friction in the compound and thermally induced/activated frictions of the GNPs particles by filler-filler and filler-matrix interaction, consistent with the improved energy dissipation observed in the vibration test.

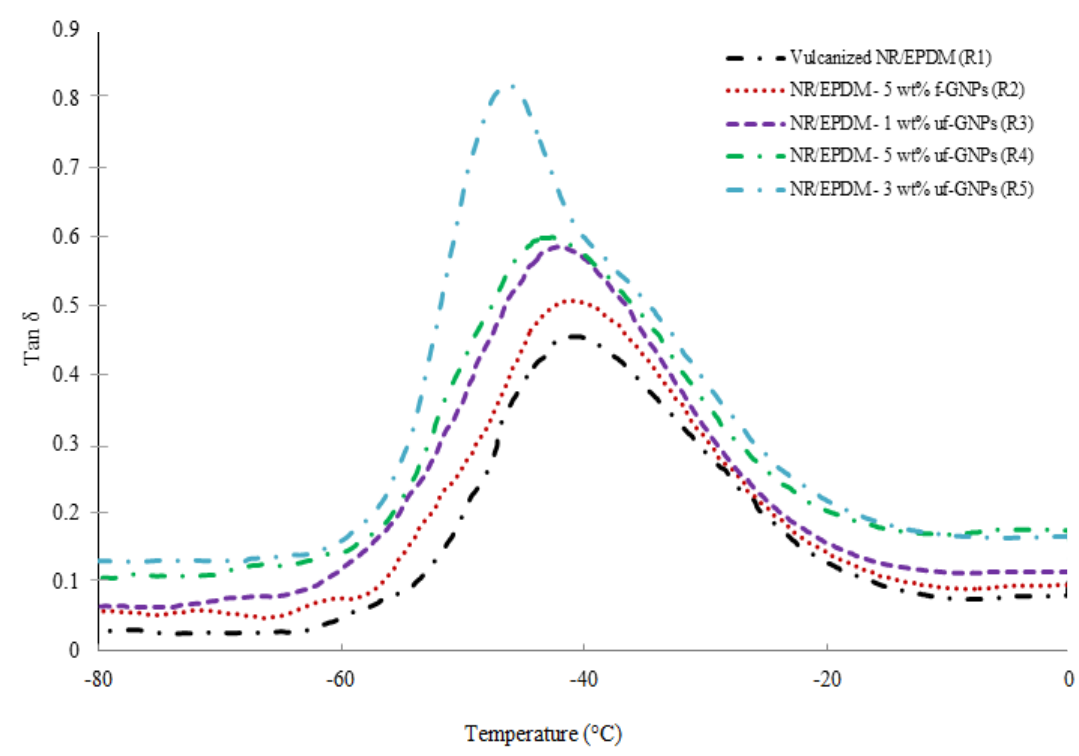

Figure 7. Tan delta as a function of temperature.

\section{Transmission Electron Microscopy}

Transmission electron microscopy was carried out to investigate the dispersion of GNPs in the NR/EPDM matrix. Figure 8 (a-d) depict the TEM micrographs of R5 (3 wt\% of unfunctionalized GNPs) and R2 (5 wt\% of functionalized GNPs). Lighter regions depict the phase with lower molecular density (EPDM phases) and darker regions represent the denser NR phases. From the TEM micrograph, R5 manifested an improved dispersion level between GNPs with NR and EPDM phases compared to R2 sample. In Figure 8 (ab), the exfoliated and intercalated state of GNP sheets between rubber particles in R5 sample are noted from the distinctive thin dark lines of only few nanometers. This indicated the separation of graphene platelets results from high shear force generated by mechanical mixing. This state of dispersion of GNPs within NR/EPDM particles assisted higher friction and mechanical interlocking in the nanocomposites structure resulting in higher damping capacity. Thus, the effective stress transfer between the matrix-GNPs interfaces improved their dynamic loading characteristics. Moreover, the arrangements of GNPs' platelet at nanoscales offered higher surface area for efficient energy absorption ensuing improvement of damping properties. However, Figure 8 (b-c) illustrate thicker and darker lines indicated the formation of GNP agglomerates and inhomogeneous distribution of GNPs within NR/EPDM matrix in R2 sample. This explained the tendency of chitosan functionalization to promote higher filler-filler interaction among the graphene platelets rather than to improve filler-matrix interaction. Therefore, morphological analysis via TEM is in good agreement with the vibration test results and dynamic mechanical analysis data. 

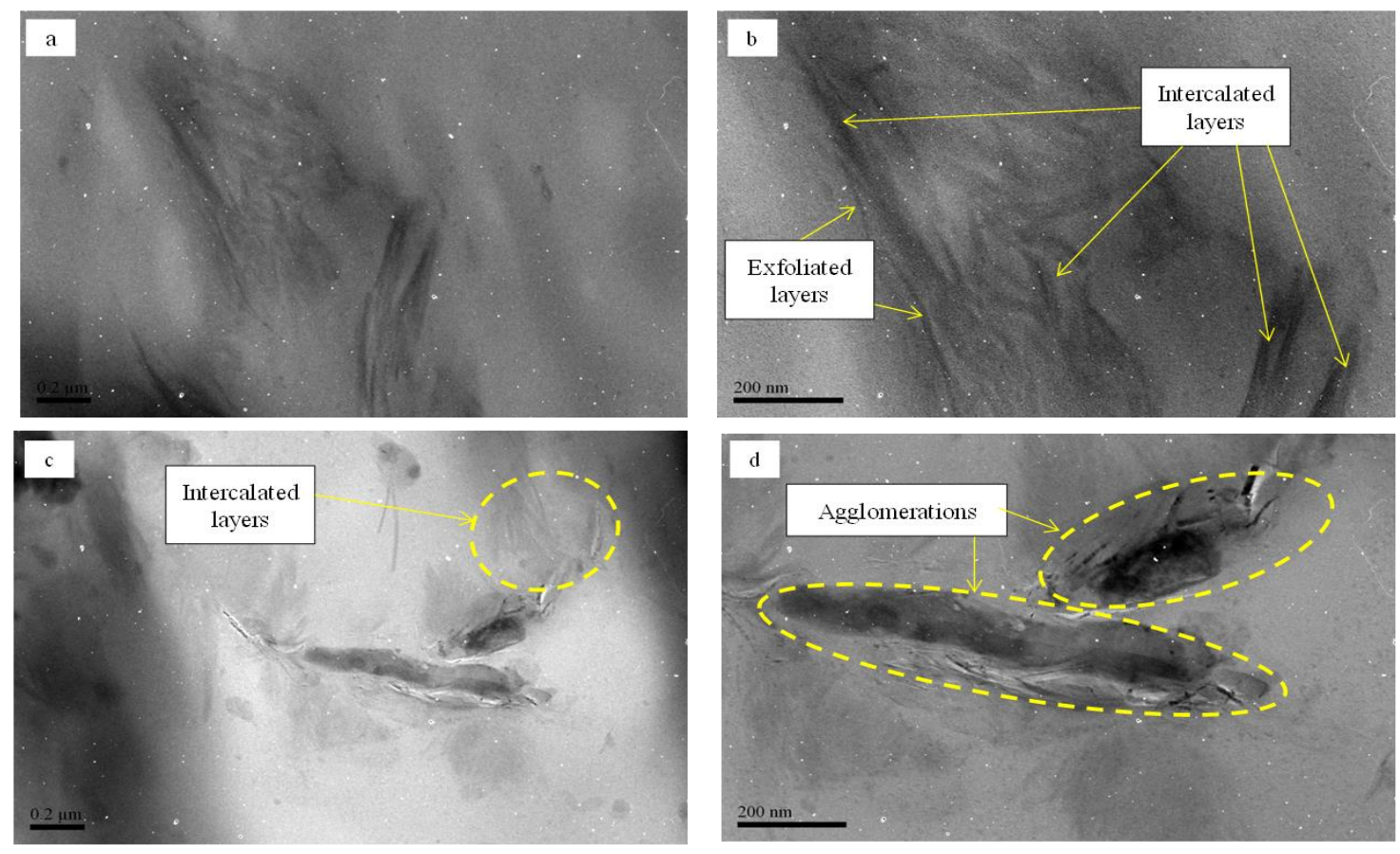

Figure 8. TEM micrographs of NR/EPDM nanocomposites (a)-(b) $3 \mathrm{wt} \%$ of unfunctionalized GNPs (R5); (c)-(d) $5 \mathrm{wt} \%$ of functionalized GNPs (R2) at different magnification.

\section{CONCLUSIONS}

The vibration damping properties of GNPs reinforced NR/EPDM nanocomposites were studied based on the free vibrations test. The vibration test via free vibration test had proven the potential of GNPs reinforced NR/EPDM as vibration isolator. From the vibration test, the addition of GNPs has significantly improved log decrement and damping ratio values up to $50.4 \%$ and $50 \%$ if compared to NR/EPDM blend. The amplitude of NR/EPDM nanocomposites with $3 \mathrm{wt} \%$ and $5 \mathrm{wt} \%$ unfunctionalized GNPs decays faster than NR/EPDM blend and those nanocomposites with lower GNPs loading and chitosan-functionalized GNPs. Their dynamic mechanical characteristics by storage modulus, loss modulus and tan $\delta$ showed good agreement with the vibrational damping behaviors measured via free vibrations test. The presence of GNPs in NR/EPDM matrix is proven to enhance the conversion of vibrational energy into heat. These results are further supported by TEM analysis which revealed the dependency of vibration damping characteristics with dispersion level and uniformity of GNPs within the NR/EPDM matrix.

\section{ACKNOWLEDGEMENT}

The authors acknowledge the Ministry of Education Malaysia and Universiti Teknikal Malaysia Melaka for funding this research through the Fundamental Research Grant Scheme (FRGS/2012/FKP/TK04/02/1 F00132) and High Impact Short Term Grant Scheme (PJP/2016/FKP/H16/S01484). We also wish to sincerely thank Malaysia Nuclear Agency which provided the expertise, equipment and technical assistance while we conducted our experiments. 


\section{REFERENCES}

[1] Gusev AA, Feldman K, Guseva O. Using elastomers and rubbers for heatconduction damping of sound and vibrations. Macromolecules 2010; 43(5): 26382641.

[2] Faiz A, Petit L, Guyomar D, et al. A new adaptive resonance frequency of piezoelectric components used for vibration damping. The Journal of the Acoustical Society of America 2010; 127(4): EL134-EL139.

[3] Singh OP, Sreenivasulu T, Kannan M. The effect of rubber dampers on engine's NVH and thermal performance. Applied Acoustics 2014; 75: 17-26.

[4] Geisberger AA, Khajepour A, Golnaraghi MF. Nonlinear modeling and experimental verification of an MDOF hydraulic engine mount. ASMEPUBLICATIONS-AD 2000; 61: 95-100.

[5] Wang J, Sun D, Liu S, Zhang X. Damping characteristics of viscoelastic damping structure under coupled condition. Mathematical and Computational Applications 2017; 22(27): doi:10.3390/mca22010027.

[6] Rosszainily IRA, Salim MA, Mansor MR, Akop MZ, Putra A, Musthafah MT, Hassan MZ, Abdul Rahman MN, Sudin MN. Effect of carbon black fillers on tensile stress of unvulcanized natural rubber compound. Journal of Mechanical Engineering and Sciences 2016; 10(2): 2043-2052.

[7] Norazlina H, Firdaus RM, Hafizuddin WM. Enhanced properties from mixing natural rubber with recycled polyvinyl chloride by melt blending. Journal of Mechanical Engineering and Sciences 2015; 8: 1440-1447.

[8] Mohamad N, Mahamood MA, Juliana Y, Jeefferie AR, Muchtar A, Shueb MI, Kassim MS, Azam MA, Yuhazri YM, Mustafa N, Toibah AR, Sahroni TR, Ahsan Q. Functionalisation of ethylene-propylene copolymer by melt grafting of maleic anhydride using a high shear internal mixer. Materials Research Innovations 2014; 18(sup6): S6-36-S6-42.

[9] Jeefferie AR, Ahmad SH, Ratnam CT, Mahamood MA, Juliana Y, Mohamad N. NR/EPDM elastomeric rubber blend miscibility evaluation by two-level fractional factorial design of experiment. AIP Conference Proceedings 2014; 1614(1): 8289.

[10] Nabil H, Ismail H, Azura AR. Comparison of thermo-oxidative ageing and thermal analysis of carbon black-filled NR/Virgin EPDM and NR/Recycled EPDM blends. Polymer Testing 2013; 32(4):631-639.

[11] Papageorgiou DG, Kinloch IA, Young RJ. Graphene/elastomer nanocomposites. Carbon 2015; 95: 460-484.

[12] Motaung TE, Luyt AS, Thomas S. Morphology and properties of nr/epdm rubber blends filled with small amounts of titania nanoparticles. Polymer Composites 2011; 32(8): 1289-1296.

[13] Razak JA, Ahmad SH, Ratnam CT, Mahamood MA, Mohamad N. Effects of poly (ethyleneimine) adsorption on graphene nanoplatelets to the properties of NR/EPDM rubber blend nanocomposites. Journal of Materials Science 2015; 50(19): 6365-6381.

[14] Kuila T, Bhadra S, Yao D, Kim NH, Bose S, Lee JH. Recent advances in graphene based polymer composites. Progress in Polymer Science 2010; 35(11): 13501375. 
[15] Kuilla T, Bose S, Mishra AK, Khanra P, Kim NH, Lee JH. Chemical functionalization of graphene and its applications. Progress in Materials Science 2012; 57(7):1061-1105.

[16] Issaadi K, Habi A, Pillin YG. Maleic anhydride-grafted poly(lactic acid) as a compatibilizer in poly(lactic acid)/graphene oxide nanocomposites. Polymer Bulletin 2016; 73: 2057.

[17] Chen C, Zhai W, Lu D, Zhang H, Zheng W. A facile method to prepare stable noncovalent functionalized graphene solution by using thionine. Materials Research Bulletin 2011; 46(4): 583-587.

[18] Mittal G, Dhand V, Rhee KY, Park SJ, Lee WR. A review on carbon nanotubes and graphene as fillers in reinforced polymer nanocomposites. Journal of Industrial and Engineering Chemistry 2015; 21: 11-25.

[19] Patole AS, Patole SP, Kang H, Yoo JB, Kim TH, Ahn JH. A Facile approach to the fabrication of graphene/polystyrene nanocomposite by in situ microemulsion polymerization. Journal of colloid and interface science 2010; 350(2): 530-537.

[20] Harish Prashanth KV, Tharanathan R. Chitin/chitosan: modifications and their unlimited application potential-An overview. Trends in food science \& technology 2007; 18(3): 117-131.

[21] Yi H, Wu LQ, Bentley WE, Ghodssi R, Rubloff GW, Culver JN, Payne GF. Biofabrication with chitosan. Biomacromolecules 2005; 6(6): 2881-2894.

[22] Ganguli S, Roy AK, Anderson DP. Improved thermal conductivity for chemically functionalized exfoliated graphite/epoxy composites. Carbon 2008; 46(5): 806817.

[23] Fang M, Long J, Zhao W, Wang L, Chen G. Ph-responsive chitosan mediated graphene dispersions. Langmuir 2010; 26(22): 16771- 16774.

[24] Juliana Y. Master of Science in Manufacturing Engineering. Universiti Teknikal Malaysia Melaka; 2015.

[25] Mohamad N, Yaakub J, Razak JA, Yaakob MY, Shueb MI, Muchtar A. Effects of epoxidized natural rubber (ENR-50) and processing parameters on the properties of NR/EPDM blends using response surface methodology. Journal of Applied Polymer Science 2014; 131(17): 40713.

[26] Khan SU, Li CY, Siddiqui NA, et al. Vibration damping characteristics of carbon fiber-reinforced composites containing multi-walled carbon nanotubes. Composites Science and Technology 2011; 71(12): 1486-1494.

[27] Kader M, Kim K, Lee YS, et al. Preparation and properties of nitrile rubber/montmorillonite nanocomposites via latex blending. Journal of Materials Science 2006; 41(22): 7341-7352.

[28] Menard KP. Dynamic mechanical analysis: a practical introduction. 2nd ed. New York: CRC press; 2008.

[29] Jindal P, Jyoti J, Kumar N. Mechanical characterisation of ABS/MWCNT composites under static and dynamic loading conditions. Journal of Mechanical Engineering and Sciences 2016; 10(3): 2288-2299.

[30] Jones DI. Handbook of viscoelastic vibration damping. England: John Wiley \& Sons; 2001. 